CLAWAR 2019: 22nd International Conference on

Climbing and Walking Robots and the Support

Technologies for Mobile Machines,

Kuala Lumpur, Malaysia, 26-28 August 2019.

https://doi.org/10.13180/clawar.2019.26-28.08.22

\title{
OPTIMIZATION OF PID CONTROLLER FOR DOUBLE-LINK FLEXIBLE ROBOTIC MANIPULATOR USING METAHEURISTIC ALGORITHMS
}

\author{
ANNISA JAMALI $\uparrow$ \\ Department of Mechanical and Manufacturing Engineering, Faculty of Engineering, \\ Universiti Malaysia Sarawak, \\ 94300 Kota Samarahan, Sarawak, Malaysia \\ E-mail: jannisa@unimas.my \\ www.unimas.my \\ INTAN ZAURAH MAT DARUS, HANIM MOHD YATIM and MAT HUSSIN BIN AB TALIB \\ Faculty of Mechanical Engineering, \\ Universiti Teknologi Malaysia, \\ 81300, Skudai, Johor, Malaysia \\ MUHAMAD SUKRI HADI \\ Faculty of Mechanical Engineering, \\ Universiti Teknologi MARA, \\ 40450, Shah Alam, Selangor, Malaysia
}

\begin{abstract}
This paper investigates the optimization approach of PID controller for double-link flexible robotic manipulator using metaheuristic algorithm. This research focus on population-based metaheuristic that is particle swarm optimization (PSO) and artificial bees algorithm (ABC) to tune the PID control parameters of the system. In the tuning process, the number of iteration was set and the number of particles was varied. The tuning process was interrupted once the convergence value of Mean Square Error (MSE) was achieved. For PSO, it was found that when the number of iteration increased, or the number of particles were set to higher values, there were no significant improvement of MSE. Results showed that 25 iterations were required for MSE to converge for hub angle and 20 iterations were required for MSE to converge for endpoint acceleration. Meanwhile, it was discovered that ABC portrayed the same pattern with PSO whereby when the number of iteration increased or the number of colony sizes were set to higher values, there were no significant improvement of MSE. From the results, 15 iterations were required for MSE to converge for hub angle and 25 iterations were required for MSE to converge for end-point acceleration. The performance of the algorithm was validated by evaluating the performance of the controllers in comparison with the conventional controller that is Ziegler Nichols (ZN) in term of input tracking capability and vibration suppression for both links. The system managed to reach desired angle for both hub angle 1 and 2 . Besides, vibration reduction shows great improvement for both link 1 and 2. This signifies that, the PSO and ABC algorithm are very effective in optimizing the PID parameters.
\end{abstract}

\section{Introduction}

The introduction of metaheuristics algorithm (MA) has brought the new avenue in the optimization problem. There are wide variety of MA. For instance, single based metaheuristics versus population based metaheuristics, local search versus global search, hybridization and memetic algorithms and parallel metaheuristics. Some of the optimization algorithm was inspired by biological processes that allow populations of organisms to adapt to their surrounding environment. These concepts were introduced by Charles Darwin back in the 19th century. The first proposal of algorithm was reported in the 60's by John Holland who introduced a Genetic Algorithm (GA). This pioneering work was the beginning of the new discovery of other optimization methods such as Differential Evolution, spiral dynamic 
algorithm, particle swarm optimization, bees algorithm, firefly, fruit fly, ant colony, bacteria foraging algorithm etc.

Optimization method has been successfully applied in many engineering problems to enhance the performance of the system including control system design. Among them, Abdullah [1] employed spiral dynamic algorithm to optimize fuzzy logic controller gains for a Unicycle Mobile Robot on irregular terrains. Simulation results showed that the optimal controller gains enable the robot to drive on the terrain and follow the predefined trajectory with the irregular profile. Lu and Liu [2] proposed ant colony to find the optimal sailing line for the use of fuzzy neural network controller of cruising vessel on the river. The recommended controller provides superior results compared to other schemes for approximately searching the shortest sailing time. Amador and Castillo [3] implemented Bee Colony Optimization method in tuning membership function of fuzzy controller for the water tank system. Simulation and the testing plant model exhibited that the parameters obtain enable the system to performance well with disturbance.

In control system design, optimization also widely used to tune the PID controller parameters'. PID controller is still the most commonly used in the industrial environment for MIMO systems because they are often capable of providing a satisfactory performance in spite of their simple structure and intuitiveness. A group of researcher has proposed a simple tuning method. There are three categories of decentralized PID control strategy that is detuning of the parameter, seeking on critical gains and obtained controllers through analytic, numerical or graphics methods by taking into accounts the interaction effects [4]. Meanwhile, more detail category has been presented in [5] that is detuning methods, sequential loop closing methods, iterative or trial-and-error methods, simultaneous solving equation or simultaneous optimization methods and independent methods.

The main issue of PID controllers is to tune the gains. Other than that, PID controller is still significant because of its robustness performance in a wide range of operating condition and easy to implement. Thus, metaheuristics algorithms have been used in various areas including in developing tuning method of PID controller for flexible manipulator. In this study dimension, Alam, et al. applied hybrid PD-PD/ILA tune by multi-objective Genetic Algorithm optimization for single link flexible manipulator (SLFM) [6]. Tijani, performed a multiobjective optimization using Differential Evolution (MODE) for PID controller of SLFM [7]. Another researcher has proposed an improved Bacterial Foraging Algorithms (BFA) to tune the PID controller of SLFM [8]. Bee Algorithm have been successful to optimize the hierarchical PID parameter of SLFM in [9]. And particle swarm optimization (PSO) algorithm to tune parameter of one PID controller of SLFM in [10]. The literatures reveal that the application of Metaheuristics Algorithm is limited to SLFM. However, the survey confirms that different type of Metaheuristics Algorithm can be used effectively in optimizing the PID controller of flexible link manipulator (FLM) system in various control strategy.

In this paper, the population based metaheuristic algorithm that is ABC and PSO are utilized to optimize all the PID controllers' gains. The validation of MA was verified via the performance of the PID controller. The optimization methods and performances validation of the tuning strategy using MA are implemented through simulation in MATLAB/Simulink environment.

\section{Metaheuristics Algorithm for PID controller Optimization}

Metaheuristics can be divided into two subcategories that is single based metaheuristics and population based metaheuristics. This research focus on population based metaheuristic that is PSO and ABC to tune the PID control parameters of the system. Both of them comprise of the global search algorithms which have the potential to find a better approximation to the solution as compared tedious conventional PID tuning method. 


\subsection{Particle Swarm Algorithm}

Particle Swarm Optimization (PSO) is developed by Kennedy and Eberhart in 1995. It is a population based stochastic optimization technique. PSO is initialized with a group of random particles or solutions and then searches for optimum by updating generations. In iteration, each particle is updated by following two "best" values. The first one is called pbest which is the best solution it has achieved so far. Another "best" value is tracked by the particle swarm optimizer and compared with pbest. This best value is a global best and called gbest. After finding the two best values, the particle updates its velocity and positions with following Eq. (1) and (2).

$$
\begin{gathered}
V_{i d}^{k+1}=W \times V_{i d}^{k}+C_{1} \times R_{1} \times\left(Y_{i d}^{k}-X_{i d}^{k}\right)+C_{2} \times R_{2} \times\left(Y_{i d}^{k}-X_{i d}^{k}\right) \\
X_{i d}^{k+1}=Y_{i d}^{k}-X_{i d}^{k}
\end{gathered}
$$

where $V=$ particle velocity, $X=$ particle position, $Y=$ gbest particle position, $W=$ Inertia weight, $R_{1}, R_{2}=$ random number and $C_{1}, C_{2}=$ learning factors. The number of particle $i$ is symbolized as the $i$-th particle in the $d$-dimensional search space and $k$ is number of iteration. Both are chosen heuristically. It was found that, the performance of optimization was significantly affected by the number of particles and the number of iteration. The cognitive part $C_{I}$ encourage the particles to move toward their own best position so far and the social component of $C_{2}$ represents the collaborative effect of the particles in finding the global optimum solution.

In this research, $C_{1}=C_{2}$ is chosen as 2 by referring to various studies of PSO in wide range of application and $R_{l}, R_{2}$ is between 0 and $1 . W$ is the inertia weight serves as memory of the previous direction, preventing the particle from drastically changing direction. The high value of $W$ promotes global exploration and exploitation, while low value of $W$ leads to a local search. The common approach is to provide balance between global and local search by linearly decreasing $W$ during the search process. The starting and end-point of inertia weight set as 0.9 and 0.25 are selected respectively in this study.

\subsection{Artificial Bee Colony}

Artificial Bee Colony $(\mathrm{ABC})$ is inspired by intelligent behavior of honey bees developed by Dervis Karaboga in in 2005. Three types of bees: employed bees, onlooker bees, and scout bees. ABC system combines local search methods, carried out by employed and onlooker bees, with global search methods, managed by onlookers and scouts, attempting to balance exploration and exploitation process.

In the $\mathrm{ABC}$ algorithm, the first half of the swarm consists of employed bees, and the second half constitutes the onlooker bees. It uses common control parameters such as colony size and maximum cycle number. The number of employed bees or the onlooker bees is equal to the number of solutions in the swarm. The $\mathrm{ABC}$ generates a randomly distributed initial population of the swarm size ( $\mathrm{SN}$ ) solutions (food sources). The initial food sources are randomly produced via the expression;

$$
x_{m}=l_{i}+\operatorname{rand}(0,1) \times\left(u_{i}-l_{i}\right)
$$


where $u_{i}$ and $l_{i}$ are the upper and lower bound of the solution space of objective function, rand $(0,1)$ is a random number within the range $[0,1]$. Each employed bee generates a new candidate solution in the neighbourhood of its present position. The neighbour food source $v_{\mathrm{mi}}$ is determined and calculated by the following equation.

$$
v_{\mathrm{mi}}=x_{\mathrm{mi}}+\varphi_{\mathrm{mi}}\left(x_{\mathrm{mi}}-x_{\mathrm{ki}}\right)
$$

where $i$ is a randomly selected parameter index, $x_{k}$ is a randomly selected food source, $\varphi_{\mathrm{mi}}$ is a random number within the range $[-1,1]$. The fitness is calculated by the following Eq. (5) after that a greedy selection is applied between $x_{m}$ and $v_{m}$.

fit $_{m}\left(x_{m}\right)=\frac{1}{1+f_{m}\left(x_{m}\right)}, f_{m}\left(x_{m}\right)>0, \quad$ fit $_{m}\left(x_{m}\right)=1+\left|f_{m}\left(x_{m}\right)\right|, f_{m}\left(x_{m}\right)<0$

where, $f_{m}\left(x_{m}\right)$ is the objective function value of $x_{m}$. The quantity of a food source is evaluated by its profitability and the profitability of all food sources. $P_{m}$ is determined by the Eq. 6);

$$
P_{m}=\frac{f i t_{m}\left(x_{m}\right)}{\sum_{m=1}^{S N} f i t_{m}\left(x_{m}\right)}
$$

where, $f i t_{m}\left(x_{m}\right)$ is the fitness of $x_{m}$. After all employed bees have completed the search processes, they share the information of their food sources with the onlooker bees through waggle dances. An onlooker bee evaluates the nectar information taken from all employed bees and chooses a food source with a probability related to its nectar amount. Onlooker bees search the neighbourhoods of food source according to the Eq. (4). If a position cannot be improved over a predefined number (called limit) of cycles, then the food source is abandoned. The scout bee discovers a new food source to replace the abandon food source. The new solution $x_{m}$ will be discovered by the scout by using the Eq. (3).

\subsection{Tuning of PID controller using PSO and ABC}

The proposed control structure using PSO and ABC were adopted to tune the PID controllers. Figures 1 and 2 show a block diagram of the closed loop system for rigid body and flexible motion control respectively. The objective functions of optimization are formulated based on the MSE of the hub angle error and end-point vibration suppression. This applied for both algorithms. The modelling of the system and the control scheme are detailed out in previous paper [11-12]

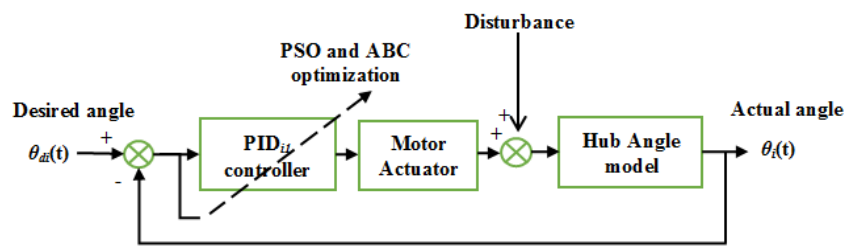

Figure 1 Block Diagram of the proposed PID control structure for hub angles 1 and 2 . 


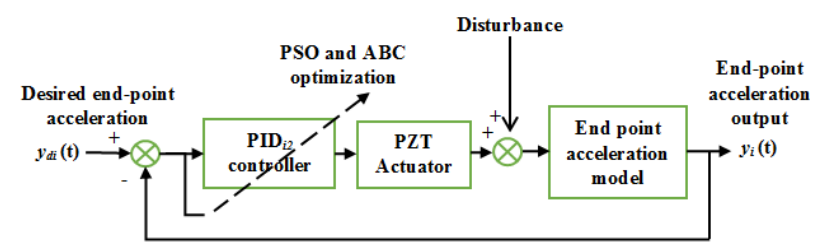

Figure 2 Block Diagram of the proposed PID control structure for end-point accelerations 1 and 2

The simulation study was conducted offline to investigate the performance of PSO and ABC algorithm to tune the PID controllers' parameters. The corresponding controller parameters which are $K_{P}, K_{I}$ and $K_{D}$ were fed to the closed-loop PID controller in MATLAB/Simulink. The error for each sample was calculated and the MSE is evaluated. MSE was set as fitness value in the algorithm. The objective is to adjust the PID controller parameters in order to minimize the fitness value.

The schematic diagram of the closed loop system utilizing PID controller with identified hub angle models and end-point vibration models are shown in Figures 3 and 4 respectively. These schematic diagrams were referred to block diagram showed in Figures 1 and 2.

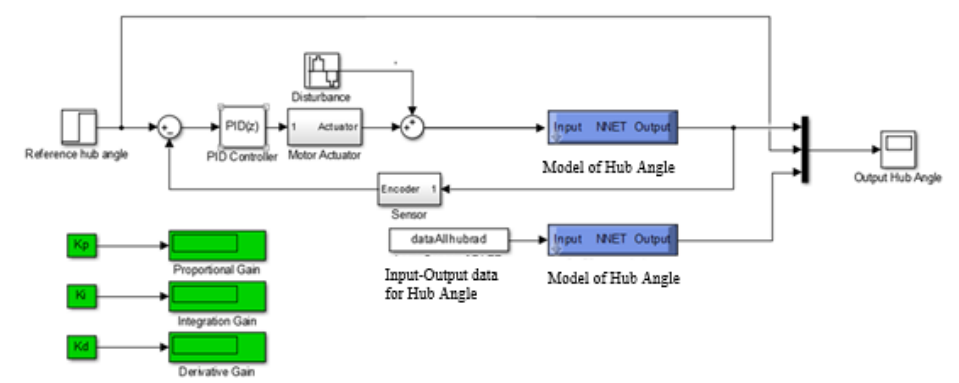

Figure 3 Simulink model for hub angle tuning by PSO and ABC

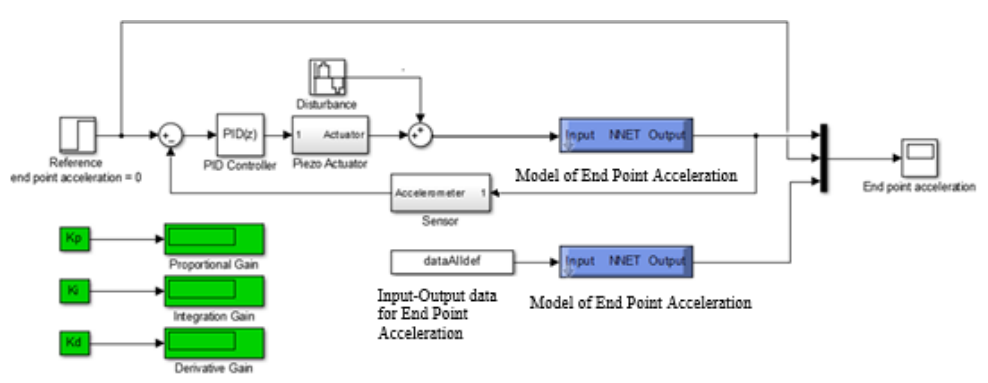

Figure 4 Simulink model for end-point acceleration tuning by PSO and ABC

Step input was used as input reference. The performance of PID controllers for hub angle models were observed in terms of $t r, t s, M p$ and Ess. Meanwhile, the performances of vibration suppression were observed in terms of the attenuation of the first three mode of vibration.

\subsection{Results and Discussion}


Results are divided into two sections that are the performance of optimization algorithms for PID controller and validation of metaheuristic algorithms.

\subsection{Performance of Optimization Algorithms for PID Controller}

The results presented the performance of PSO and ABC to tune the PID parameters' based on MSE value.

\subsubsection{PSO}

In this study, the number of particles and number of iterations were tuned heuristically. The tuning is initialized by setting the number of iterations to 15 and varying the number of particles from 10 to 50. The same procedure was repeated for 50 maximum iterations. For PSO, satisfactory result was obtained with 20 particles at 25 th iteration for both hub angle and 50 particles at 30th iteration for end-point acceleration suppression. The PID controllers were evaluated based on the minimum value of MSE. It was discovered that, when the number of iteration increased, or the number of particles were set to higher values, there were no significant improvement of MSE. Figures 5 (a) and (b) show the 25 iterations of MSE convergence of PSO for hub angle. Meanwhile, Figure 6 (a) and (b) show the 20 iterations of MSE convergence of PSO for end-point acceleration. It can be observed that PSO optimization converges quite fast and produce small value of MSE for all the four controllers. The convergence MSE values with respect to the PID parameters obtained are tabulated in Table 1.

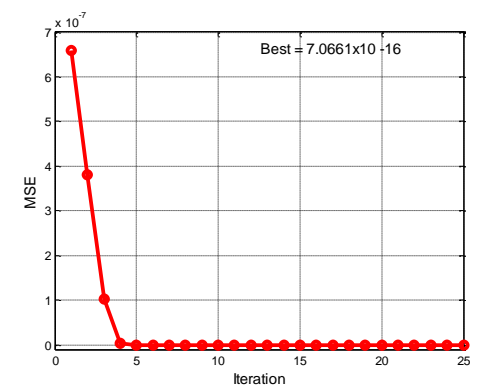

(a) Link 1

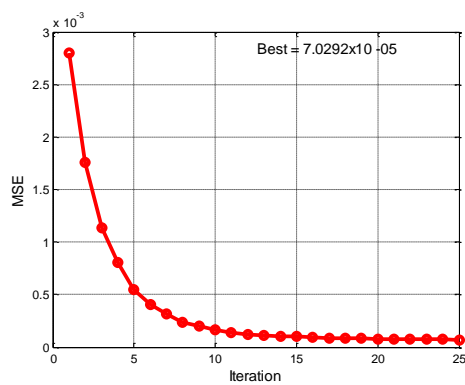

(b) Link 2

Figure 5 PSO convergence hub angle

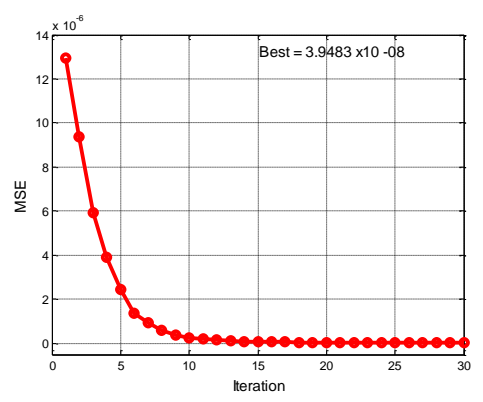

(a) Link 1

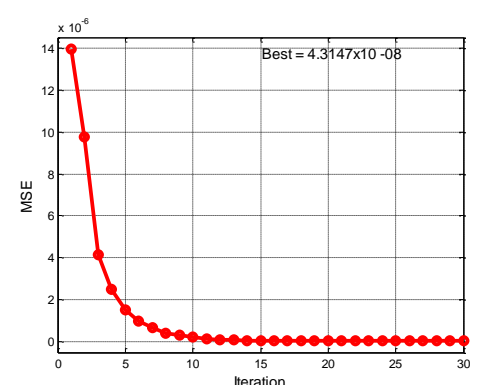

(b) Link 2

Figure 6 PSO convergence for end-point acceleration 
Table 1: Convergence of MSE for PSO Algorithm

\begin{tabular}{cccccc}
\hline \multirow{2}{*}{ Type of Algorithm } & Parameter & MSE & \multicolumn{3}{c}{ PID Parameters } \\
\cline { 3 - 6 } & Hub Angle 1 & $7.0661 \times 10^{-16}$ & 3.65 & 57.9 & 3.46 \\
\hline \multirow{2}{*}{ PSO } & End of Acceleration 1 & $3.9483 \times 10^{-08}$ & 2.07 & 498.1 & 2.04 \\
& Hub Angle 2 & $7.0292 \times 10^{-05}$ & 2.19 & 88.2 & 0.79 \\
& End of Acceleration 1 & $4.3147 \times 10^{-08}$ & 8.06 & 817.9 & 1.03
\end{tabular}

\subsection{2 $\mathrm{ABC}$}

In this study, the number of colony size and number of iterations were tuned heuristically. The tuning is initialized by setting the number of iterations to 15 and varying the number of colony size from 10 to 50. The same procedure was repeated for 50 maximum iterations. For ABC, satisfactory result was obtained with 50 colony sizes at 15 th iteration for both hub angle and 50 colony sizes at 25th iteration for end-point acceleration suppression. The PID controllers were evaluated based on the minimum value of MSE. It was discovered that, when the number of iteration increased or the number of colony sizes were set to higher values, there were no significant improvement of MSE. Figure 7 (a) and (b) show the 15 iterations of MSE convergence of $\mathrm{ABC}$ for hub angle. Meanwhile, Figure 8 (a) and (b) show the 25 iterations of MSE convergence of $\mathrm{ABC}$ for end-point acceleration. It can be observed that $\mathrm{ABC}$ optimization converges quite fast and produce small value of MSE for all the four controllers. The convergence MSE values with respect to the PID parameters obtained are tabulated in Table 2.

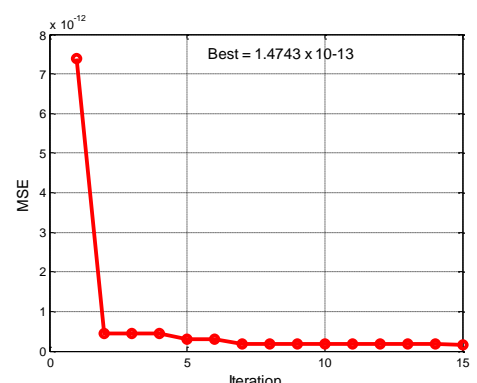

(a) Link 1

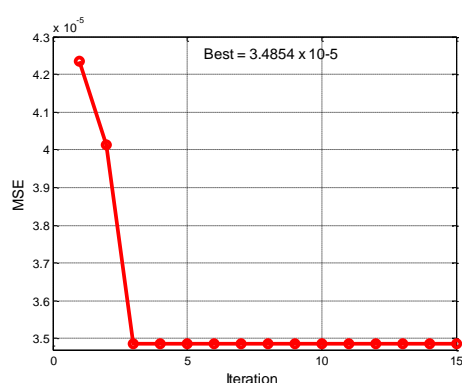

(b) Link 2

Figure $7 \mathrm{ABC}$ convergence for hub angle

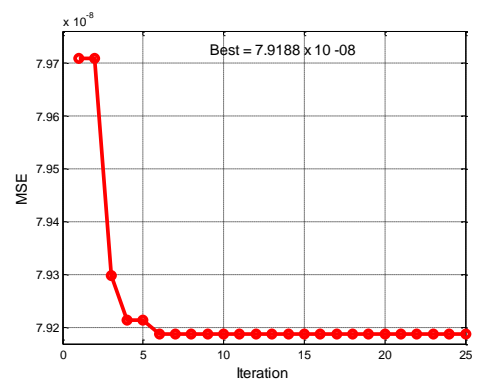

(a) Link 1

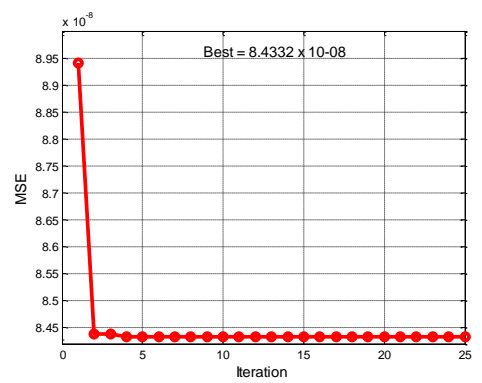

(b) Link 2

Figure $8 \mathrm{ABC}$ convergence for end-point acceleration 
Table 2: Convergence of MSE for ABC Algorithm

\begin{tabular}{cccccc}
\hline \multirow{2}{*}{ Type of Algorithm } & Marameter & \multicolumn{3}{c}{ PID Parameters } \\
\cline { 3 - 6 } & Hub Angle 1 & $1.4743 \times 10^{-13}$ & 6.54 & 20.5 & 49.43 \\
& End of Acceleration 1 & $7.9188 \times 10^{-08}$ & 30.03 & 56.07 & 88.95 \\
\multirow{2}{*}{ ABC } & Hub Angle 2 & $3.4854 \times 10^{-05}$ & 5.48 & 28.3 & 13.72 \\
& End of Acceleration 1 & $8.4332 \times 10^{-08}$ & 50.1 & 46.96 & 23.62 \\
\hline
\end{tabular}

\subsection{Validation of Metaheuristics Algorithm via the performance of the controllers}

The performance of the algorithm was validated by evaluating the performance of the controllers in comparison with the conventional controller that is Ziegler Nichols (ZN). Results of the simulation are divided into two sections that are hub angle control and flexible motion control. For hub angle control, the outcomes were measured in term of input tracking capability. Meanwhile, for vibration the outcomes were assessed via the suppression level of vibration in both links.

\subsubsection{Hub angle control}

The hub angles were controlled by the tuned PID controller individually. The DLFRM system is required to follow a step input of $2.1 \mathrm{rad}$ and $1.1 \mathrm{rad}$ to test the hub tracking input of link 1 and 2 respectively. The hub angle response for both link is shown in Figure 9. PSO and ABC controller achieved a very significance improvement in term rise time, steady state error and overshoot in comparison to Ziegler-Nichols (ZN). Table 3 exhibits the tabulated results for those parameters.

Overall, the results showed PSO and ABC-based controller supersede Ziegler-Nichols (ZN) controller in all aspect of system response. In brief, both the PSO and ABC-based control used in this work achieved satisfactory hub angle response.

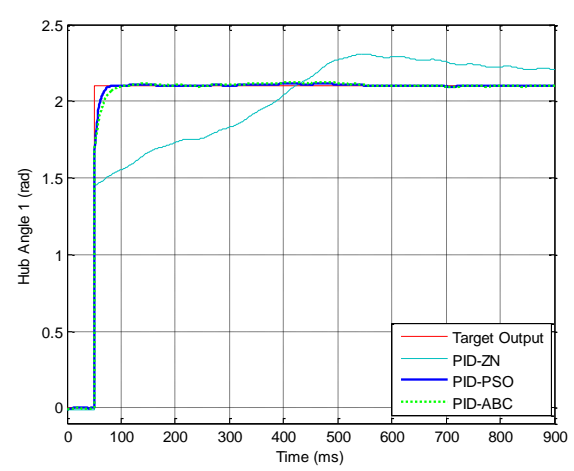

(a)

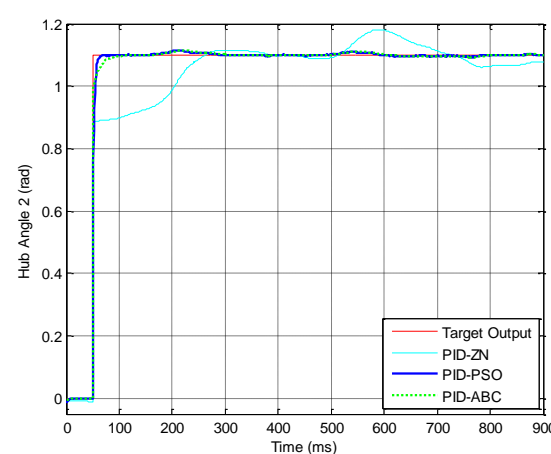

(b)

Figure 9: (a) Input tracking for Hub 1 (b) Input tracking for Hub 2 
Table 3: Parameters and Performance of hub input tracking for DLFR system

\begin{tabular}{ccccccccc}
\hline & & \multicolumn{3}{c}{ Parameters } & Rise Time (s) & Settling Time (s) & Overshoot (\%) & SSE \\
& & $K_{P}$ & $K_{I}$ & $K_{D}$ & & & & \\
\hline ABC & L 1 & 6.54 & 20.5 & 49.43 & 0.076 & 1.08 & 1.94 & 0.007 \\
& L 2 & 5.48 & 28.3 & 13.72 & 0.099 & 5.64 & 3.19 & 0.002 \\
PSO & L1 & 3.65 & 57.9 & 3.46 & 0.058 & 1.16 & 0.89 & 0.003 \\
& L 2 & 2.19 & 88.2 & 0.79 & 0.043 & 0.59 & 1.64 & 0.002 \\
ZN & L1 & 2.09 & 0.54 & 2.01 & 2.97 & 7.15 & 4.69 & 0.681 \\
& L2 & 4.15 & 1.3 & 3.32 & 1.46 & 5.45 & 5.45 & 0.284 \\
\hline
\end{tabular}

\subsubsection{Flexible motion control}

The tuned PID controllers using both metaheuristic algorithms were implemented to DLFRM system to actively suppress the vibration at the end point of link 1 and 2 individually. The simulation results of vibration suppression are presented in Figure 10. It can be observed from Figure 7 that the responses from ABC and PSO tuning methods are having almost the same amplitude of vibration. In general, both metaheuristics algorithm used to tune the PID controller achieved satisfactory vibration suppression.

Table 4 displays the tabulated results of attenuation value for both algorithms. There are more than $70 \%$ improvement of vibration suppression as compared to conventional tuning method. In conclusion, PSO and ABC-based control can provide a better suppression in comparison to Ziegler-Nichols (ZN).

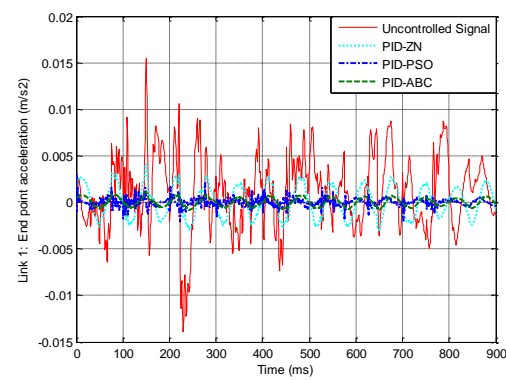

(a)

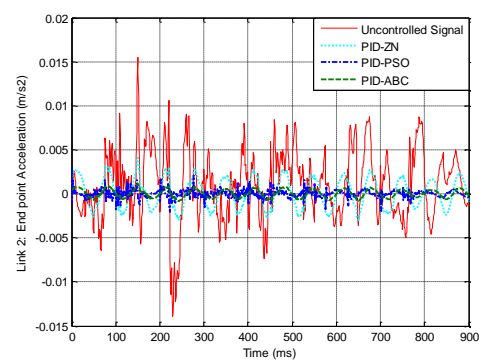

(b)

Figure 10: (a) End point vibration suppression of Hub 1(b) End point vibration suppression of Hub 2

Table 4: Parameters and Performance of vibration suppression for DLFR system.

\begin{tabular}{|c|c|c|c|c|c|c|c|c|}
\hline & & \multicolumn{3}{|c|}{ Parameters } & \multirow[t]{2}{*}{ MSE } & \multicolumn{3}{|c|}{ Attenuation of amplitude at natural frequency $(\mathrm{dB})$} \\
\hline & & $K_{P}$ & $K_{I}$ & $K_{D}$ & & $1^{\text {st }}$ & $2^{\text {nd }}$ & $3^{\text {rd }}$ \\
\hline \multirow[t]{2}{*}{$\mathrm{ABC}$} & L1 & 30.03 & 56.07 & 88.95 & $7.919 \mathrm{e}-07$ & 35.27 & 67.7 & 67.6 \\
\hline & L2 & 50.1 & 46.96 & 23.62 & $8.432 \mathrm{e}-08$ & 39.8 & 82.2 & 83.4 \\
\hline \multirow[t]{2}{*}{ PSO } & L1 & 2.07 & 498.1 & 2.04 & $3.948 \mathrm{e}-08$ & 45.77 & 27 & 12 \\
\hline & $\mathrm{L} 2$ & 8.06 & 817.9 & 1.03 & $4.315 \mathrm{e}-08$ & 43.3 & 44.4 & 32.6 \\
\hline \multirow[t]{2}{*}{$\mathrm{ZN}$} & L1 & 7.2 & 21.176 & 0.612 & $2.822 \mathrm{e}-06$ & 8.9 & 41 & 40.9 \\
\hline & $\mathrm{L} 2$ & 16 & 55.082 & 1.281 & $7.564 \mathrm{e}-07$ & 11.8 & 53.3 & 54.4 \\
\hline
\end{tabular}

\section{$5.0 \quad$ Conclusions}

In this work, the optimization approach of PID controller using metaheuristic algorithm is carried out. This research focus on population based metaheuristic that is PSO and ABC to tune the PID control parameters of double-link flexible robotic manipulator. The validation was employed via the performance of the PID controllers of DLFRM system. Results showed that PSO and ABC-based control can achieve satisfactory hub angle response and provide a better suppression in comparison to Ziegler-Nichols (ZN). 


\section{Acknowledgments}

The authors would like to express their gratitude to Minister of Education Malaysia (MOE), Universiti Malaysia Sarawak (UNIMAS) and Universiti Teknologi Malaysia (UTM) for funding (Vote No: 4J388) and providing facilities to conduct this research.

\section{References}

1. Abdullah M. Almeshal, M. R. A., and H.Alzanki, T. (2014). A Spiral Dynamic Optimised Hybrid Fuzzy Logic Controller for Unicycle Mobile Robot on Irregular Terrains. World Academy of Science, Engineering and Technology, (10), 1508-1512.

2. Lu, H. and Liu, H. (2013). Ant colony fuzzy neural network controller for cruising vessel on river. Applied Ocean Research, 42, 43-54.

3. Amador-angulo, L. and Castillo, O. (2014). Optimization of the Type-1 and Type-2 Fuzzy Controller design for the Water Tank using the Bee Colony Optimization. In Norbert Wiener in the 21 st Century, 1-8

4. Vázquez, F., \& Morilla, F., "Tuning decentralized PID controllers for MIMO systems with decouplers". In Proceedings of the 15th IFAC World Congress, (2002).

5. Huang, H. P., Jeng, J. C., Chiang, C. H., \& Pan, W., "A direct method for multi-loop PI/PID controller design”, Journal of Process Control, (2003), Vol.13, 769-781.

6. Alam, M. S., Md Zain, M. Z., Tokhi, M. O., \& Aldebrez, F., "Design of hybrid learning control for flexible manipulators: A multi-objective optimisation approach," in Proceedings of CLAWAR, 2006, pp. 599-606.

7. Tijani, I. B., Akmeliawati, R., Muthalif, A. G. A., \& Legowo, A., "Optimization of PID controller for flexible link system using a pareto-based multi-objective differential evolution," in Proceeding of 4th International Conference on Mechatronics, 2011.

8. Supriyono, H., Tokhi, M. O., \& Md Zain, B. A., "Control of a single-link flexible manipulator using improved bacterial foraging algorithm," in Proceeding of Conference on Open Systems, 2010, pp. 68-73.

9. Fahmy, A. A., Kalyoncu, M., \& Castellani, M., "Automatic design of control systems for robot manipulators using the bees algorithm," Journal of Systems and Control Engineering, vol.1, pp. 1-12, 2011.

10. Yatim, H., \& Mat Darus, I. Z., "Self-tuning Active Vibration Controller using Particle Swarm Optimization for Flexible Manipulator System", WSEAS Transactions on Systems and Control, 2014, Vol.9, 55-66.

11. J. Annisa, I.Z. Mat Darus1, M.O. Tokhi, P. P. Mohd Samin (2018). Intelligent Modeling of Double Link Flexible Robotic Manipulator Using Artificial Neural Network, Journal of Vibroengineering (Vol. 20, Issue 3), ISSN Print 1392-8716.

12. J. Annisa, I. Z. M. Darus, M. O. Tokhi and S. Mohamaddan, "Implementation of PID Based Controller Tuned by Evolutionary Algorithm for Double Link Flexible Robotic Manipulator," 2018 International Conference on Computational Approach in Smart Systems Design and Applications (ICASSDA), Kuching, 2018, pp. 1-5. 